\title{
Raman Spectroscopic Studies on Two-Dimensional Materials
}

\author{
Jae-Ung Lee, Minjung Kim, Hyeonsik Cheong* \\ Department of Physics, Sogang University, Seoul 04107, Korea
}

\author{
*Correspondence to: \\ Cheong $\mathrm{H}$, \\ Tel: +82-2-705-8434 \\ Fax: +82-2-717-8434 \\ E-mail: hcheong@sogang.ac.kr \\ Received September 11, 2015 \\ Revised September 13, 2015 \\ Accepted September 13, 2015
}

\begin{abstract}
Raman spectroscopy is one of the most widely used tools in the field of graphene and two-dimensional (2D) materials. It is used not only to characterize structural properties such as the number of layers, defect densities, strain, etc., but also to probe the electronic band structure and other electrical properties. As the field of $2 \mathrm{D}$ materials expanded beyond graphene to include new classes of layered materials including transition metal dichalcogenides such as $\mathrm{MoS}_{2}$, new physical phenomena such as anomalous resonance behaviors are observed. In this review, recent results from Raman spectroscopic studies on 2D materials are summarized.
\end{abstract}

Key Words: Raman spectroscopy, Two-dimensional materials, Transition metal dichalcogenides

\section{INTRODUCTION}

Recently, two-dimensional (2D) materials attract much interest thanks to their unique physical properties and possible device applications. Graphene, a single atomic layer of graphite, is representative of 2D materials which have been studied intensively for about 10 years (Novoselov et al., 2004; Geim \& Novoselov, 2007). Recently, the field has expanded to other layered materials, such as hexagonal boron nitride (Dean et al., 2010), transition metal dichalcogenides (TMDCs) (Mak et al., 2010; Wang et al., 2012), black phosphorus (Li et al., 2014; Liu et al., 2014), and so on. The physical properties of such layered materials vary greatly, from metals to semiconductors to insulators, and even some superconductors. In layered materials, each layer is weakly bonded by van der Waals interactions, and so the layers are easily exfoliated as shown in Fig. 1, which allows investigation of the properties of few-layer materials as opposed to bulk properties. In fewlayer materials, the physical properties are strongly dependent on the number of layers since the interlayer interactions, albeit fairly small, play an important role in determining the band structure and other physical properties (Castro Neto et al., 2009). Therefore, identifying the number of layers has been a key issue in studying these materials. Although various techniques have been used to identify the number of layers of the 2D materials, Raman spectroscopy remains one of the most widely used characterization methods (Ferrari \& Basko, 2013). In this Review, we will summarize recent progress in Raman spectroscopy of graphene and other 2D materials.

\section{DETERMINATION OF NUMBER OF LAYERS}

In the case of graphene, the correlation between the Raman spectrum and the number of layers was established early on (Ferrari et al., 2006; Yoon et al., 2009a) and is still extensively used. The line shape of the so-called 2D band is strongly dependent on the number of layers, especially for 1 to 5 layers. More recently, this technique has expanded to identifying different stacking orders in graphene (Cong et al., 2011; Lui et al., 2011; Nguyen et al., 2014). Few-layer graphene can have two types of stacking, the usual Bernal or ABA stacking and rhombohedral or $\mathrm{ABC}$ stacking. The line shape of the $2 \mathrm{D}$ band depends not only on the number of layers, but also on the stacking order and the excitation energy of the laser used in the Raman measurement. By choosing appropriate excitation energies, one can unambiguously determine both

This work was supported by the National Research Foundation (NRF) grants funded by the Korean government (MSIP) (Nos. 2011-0013461 and 2011-0017605) and by a grant (No. 2011-0031630) from the Center for Advanced Soft Electronics under the Global Frontier Research Program of MSIP.

@ This is an open-access article distributed under the terms of the Creative Commons Attribution Non-Commercial License (http://creativecommons.org/licenses/by-nc/4.0) which permits unrestricted noncommercial use, distribution, and reproduction in any medium, provided the original work is properly cited.

Copyrights @ 2015 by Korean Society of Microscopy 


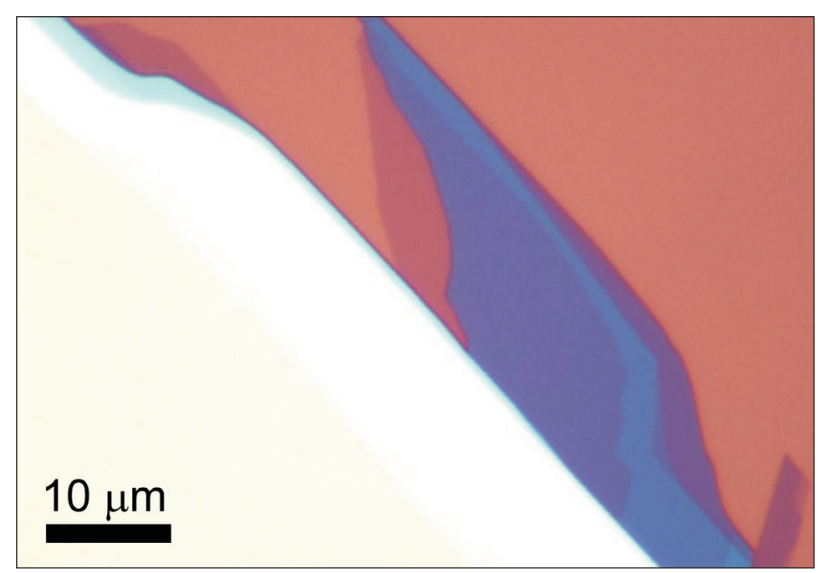

Fig. 1. Optical microscope image of a few-layer $\mathrm{MoS}_{2}$ sample on a $\mathrm{SiO}_{2} / \mathrm{Si}$ substrate.

the number of layers and the stacking order.

In the case of $\mathrm{MoS}_{2}$, the most extensively studied among semiconducting TMDCs, the separation between the two main modes $\left(\mathrm{E}_{2 \mathrm{~g}}{ }^{1}\right.$ and $\mathrm{A}_{1 \mathrm{~g}}$ modes) have been used to identify the number of layers (Lee et al., 2010). Fig. 2 shows typical Raman spectra of 1 to 4 layer $\mathrm{MoS}_{2}$. The $\mathrm{E}_{2 \mathrm{~g}}{ }^{1}$ mode corresponds to in-plane vibrations, and the $\mathrm{A}_{1 \mathrm{~g}}$ mode to outof-plane vibrations. The $\mathrm{E}_{2 \mathrm{~g}}{ }^{1}$ mode redshifts as the number of layers increases whereas the $\mathrm{A}_{1 \mathrm{~g}}$ mode blue shifts (Lee et al., 2010). The blue shift of $A_{1 g}$ mode with the number of layers can be explained by classical coupled harmonic oscillator, whereas the redshift of $\mathrm{E}_{2 \mathrm{~g}}{ }^{1}$ with increasing number of layers has been attributed to the enhancement of dielectric screening of the long-range Coulomb interactions (Lee et al., 2010; Molina-Sánchez \& Wirtz, 2011). Similar dependence of the $\mathrm{E}_{2 \mathrm{~g}}{ }^{1}$ and $\mathrm{A}_{\mathrm{lg}}$ modes on the number of layers has been observed for other semiconducting TMDCs such as $\mathrm{MoSe}_{2}$ (Tonndorf et al., 2013; Chen et al., 2015), WS (Zhao et al., 2013a; Chen et al., 2015), and $\mathrm{WSe}_{2}$ (Luo et al., 2013; Zhao et al., 2013a; Chen et al., 2015). Furthermore, in $\mathrm{MoSe}_{2}$ and $\mathrm{WS}_{2}$, splitting of the $A_{1 g}$ mode has been observed (Tonndorf et al., 2013; Chen et al., 2015; Staiger et al., 2015). This splitting is known as the Davydov splitting due to interlayer interactions. Since the number of Raman active modes among such Davydov split modes and their frequencies depend on the number of layers, the number of layers can be reliably determined by monitoring the Davydov splitting (Tonndorf et al., 2013; Staiger et al., 2015). In the case of $\mathrm{WSe}_{2}, \mathrm{E}_{2 \mathrm{~g}}{ }^{1}$ and $\mathrm{A}_{1 \mathrm{~g}}$ modes are closely overlapped and difficult to distinguish. Polarized Raman measurements can resolve this issue. Depending on the symmetry of vibrations, the polarization selection rule for each mode is different. By using linearly polarized light, the $\mathrm{E}_{2 \mathrm{~g}}{ }^{1}$ mode can be observed for both parallel and cross polarization configurations, whereas the $A_{1 g}$ mode can be seen only in parallel polarization (Zhao et al., 2013b). A more

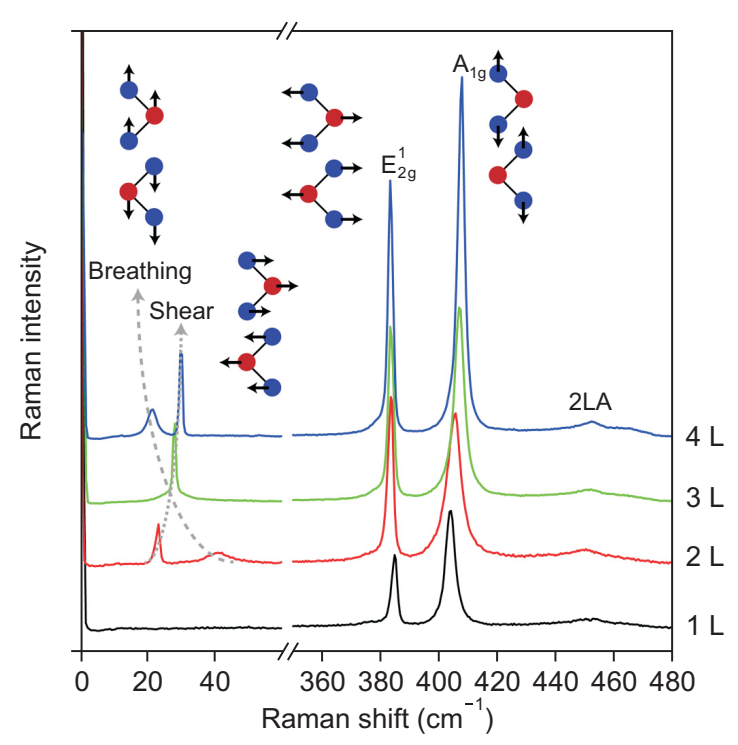

Fig. 2. Raman spectra of 1 to 4-layer $\mathrm{MoS}_{2}$ measured with the excitation energy of $2.41 \mathrm{eV}$. Vibrational modes are shown schematically.

efficient way to distinguish these modes is using circularly polarized light. If the helicity of the incident and the scattered light is same, only the $A_{1 g}$ mode is observed, whereas only the $\mathrm{E}_{2 \mathrm{~g}}{ }^{1}$ modes is observed with different helicity (Chen et al., 2015). This is an effective way to measure the frequency difference between these two modes especially for $\mathrm{WSe}_{2}$. Even though the main modes are good indicator of the number of layers, there are several limitations. Since the incremental shift gets smaller as the number of layers increases, this technique is limited to $\sim 4$ layers. In addition, because these modes are sensitive to other effects such as strain, doping, and the substrate, incorrect determination is possible. A more definitive way to determine the number of layers is by comparing the interlayer shear and breathing modes (Tan et al., 2012; Zhang et al., 2013; Zhao et al., 2013b; Lee et al., 2015; Zhang et al., 2015). These vibration modes are schematically shown in Fig. 2. They are rigid vibrations of the layers along the in-plane and out-of-plane directions. Most of few-layer 2D crystals are expected to have these vibrational modes. Since these modes shift sensitively with the number of layers, they are good fingerprints of the number of layers. On the negative side, theses vibrations are located in the lowfrequency region below $100 \mathrm{~cm}^{-1}$, which makes it difficult to observe experimentally. A triple stage spectrometer or a single-stage spectrometer with recently developed Bragg notch filters should be used to access Raman spectra in this frequency range (Tan et al., 2012; Lee et al., 2015). The shear and breathing modes of several 2D materials have been reported (Tan et al., 2012; Zhang et al., 2013; Zhao et al., 2013b; Chen et al., 2015; Lee et al., 2015). In the case of $\mathrm{MoS}_{2}$, the number of layers can be identified up to 14 layers by using 
the shear and breathing modes (Lee et al., 2015).

\section{EFFECT OF EXCITATION ENERGY}

For Raman measurements of 2D materials, choosing the right excitation energy is an important factor. For example, the optical interference effect for $2 \mathrm{D}$ materials on $\mathrm{SiO}_{2} / \mathrm{Si}$ substrates greatly affects the observed Raman intensity (Yoon et al., 2009b). It is well known that in order to enhance the visibility of a sample on $\mathrm{SiO}_{2} / \mathrm{Si}$ substrates under the optical microscope (Blake et al., 2007), optimal $\mathrm{SiO}_{2}$ thickness of 90 and $285 \mathrm{~nm}$ should be used. Similar interference effects also affect the Raman intensity of the 2D materials (Yoon et al., 2009b; Li et al., 2012). For thicker samples, the optimal laser wavelength depends on both the thickness of the sample and that of the $\mathrm{SiO}_{2}$ layer on the substrate.

When the excitation energy matches an allowed optical transition energy, resonance Raman scattering occurs. Fig. 3 shows the effect of resonance on the Raman spectrum of $\mathrm{MoS}_{2}$. In semiconducting TMDCs, complicated excitonic structures have been expected due to reduced dielectric screening of Coulomb interactions in 2D crystals (Qiu et al., 2013; Klots et al., 2014). Due to extremely large exciton binding energies, resonances with such exciton states exhibit several anomalous behaviors as reported by several groups (Chakraborty et al., 2013; Fan et al., 2014; Gołasa et al., 2014; Carvalho et al., 2015; Lee et al., 2015; Livneh et al., 2015; Pimenta et al., 2015). When the excitation energy matches the A or B exciton state, many second order Raman peaks are enhanced due to resonance with the exciton state. These second order peaks have been assigned to combinations of two phonon modes at the zone boundary near the $\mathrm{M}$ or $\mathrm{K}$ point in the Brillouin zone (Fan et al., 2014; Gołasa et al., 2014; Lee et al., 2015). However, still several peaks remain unassigned and the exact mechanism of such enhancement is not clearly understood yet. Further theoretical and

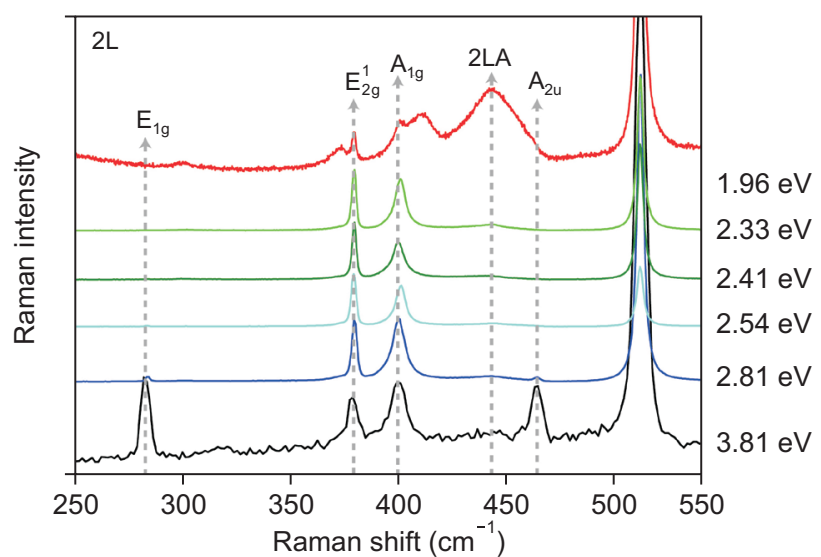

Fig. 3. Raman spectra of 2-layer $\mathrm{MoS}_{2}$ measured with 6 excitation energies. experimental studies are needed. In $\mathrm{MoS}_{2}$, the broad peak centered at the Rayleigh scattered laser line appears when a laser $(1.96 \mathrm{eV})$ that matches the exciton energy is used, due to resonance excitation of the exciton states mediated by acoustic phonon scattering (Lee et al., 2015). This peak appears broader and stronger for single-layer $\mathrm{MoS}_{2}$, which suggests that the inhomogeneity due to substrate is more pronounced in single-layer $\mathrm{MoS}_{2}$. The intensities of the main $\mathrm{E}_{2 \mathrm{~g}}{ }^{1}$ and $\mathrm{A}_{1 \mathrm{~g}}$ modes also change with the excitation energy. In $\mathrm{MoS}_{2}$, the $\mathrm{A}_{1 \mathrm{~g}}$ mode is enhanced when the excitation energy matches the $A$ and B exciton states (Scheuschner et al., 2012; Carvalho et al., 2015). However the $\mathrm{E}_{2 \mathrm{~g}}{ }^{1}$ mode shows a different behavior. The $\mathrm{E}_{2 \mathrm{~g}}{ }^{1}$ mode is relatively enhanced at a higher excitation energy of $2.81 \mathrm{eV}$, which is close to the C exciton state in $\mathrm{MoS}_{2}$ (Qiu et al., 2013; Klots et al., 2014). The selective enhancement of the $\mathrm{E}_{2 \mathrm{~g}}{ }^{1}$ mode with respect to the $\mathrm{A}_{1 \mathrm{~g}}$ mode has been explained by symmetry dependent exciton-phonon coupling (Carvalho et al., 2015). In the case of $\mathrm{WSe}_{2}$, different resonance behaviors for first- and second-order Raman modes are reported (del Corro et al., 2014). The first-order Raman modes are only weakly enhanced at resonance with the B exciton state but are strongly enhanced at resonance with $\mathrm{A}^{\prime}$ and $\mathrm{B}^{\prime}$ excited exciton states. Second-order Raman modes are enhanced at resonance with both $\mathrm{B}$ exciton and the excited exciton states in contrast to first-order Raman modes. This difference has been attributed to different electron-phonon coupling (del Corro et al., 2014).

\section{EFFECTS OF STRAIN, DOPING, AND DEFECT}

In addition to determination of the number of layers, Raman spectroscopy has been used to probe other basic characteristics of graphene such as defects (Dresselhaus et al., 2010; Cançado et al., 2011; Eckmann et al., 2012), strain (Mohiuddin et al., 2009; Yoon et al., 2011), and doping (Yan et al., 2007; Lee et al., 2012). Furthermore, the crystallographic orientation can be determined by measuring the polarization dependence of the Raman spectrum with the sample under uniaxial strain (Mohiuddin et al., 2009; Yoon et al., 2011; Jegal et al., 2013). For $\mathrm{MoS}_{2}$, several groups have reported Raman measurements under uniaxial strain (Conley et al., 2013; Wang et al., 2013). The in-plane $\mathrm{E}_{2 \mathrm{~g}}{ }^{1}$ mode splits and redshifts whereas the outof-plane $A_{1 g}$ mode is not affected by uniaxial strain. As in the case of graphene, the split $\mathrm{E}_{2 \mathrm{~g}}{ }^{1}$ modes exhibit orthogonal polarization behaviors. From the polarization dependence of the split $\mathrm{E}_{2 \mathrm{~g}}{ }^{1}$ modes, the crystallographic orientation with respect to the strain axis can be determined (Wang et al., 2013). The $A_{1 g}$ mode of $M_{2} S_{2}$ is sensitive to doping. When a gate voltage is applied to induce electron doping in $\mathrm{MoS}_{2}$, redshift and broadening of the $\mathrm{A}_{1 \mathrm{~g}}$ mode are observed whereas the $\mathrm{E}_{2 \mathrm{~g}}{ }^{1}$ mode is not affected (Chakraborty et al., 2012). This is due to stronger electron-phonon coupling of the $A_{1 g}$ mode. 


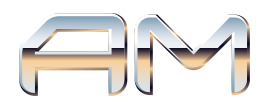

Therefore, the $\mathrm{A}_{1 \mathrm{~g}}$ mode can be used to probe the effect of doping in $\mathrm{MoS}_{2}$ (Chakraborty et al., 2012). Furthermore, the density of defects in $\mathrm{MoS}_{2}$ can be estimated through Raman spectroscopy (Mignuzzi et al., 2015). By comparing the Raman spectra of ion bombarded $\mathrm{MoS}_{2}$ samples with that of pristine samples, the effect of disorder on the Raman spectrum was investigated (Mignuzzi et al., 2015). Both the evolution of the linewidths and the frequency shift of the main first-order Raman modes with increasing density of defects are explained in terms of the phonon confinement model. Several peaks corresponding to zone boundary phonons are activated as the density of defects increases. The LA(M) mode at $\sim 227 \mathrm{~cm}^{-1}$ is most pronounced, and its relative intensity with respect to the first-order Raman modes is proportional to the density of defects. Therefore, quantitative estimation of the density of defects is possible by using the intensity of LA(M) mode as the D peak intensity is used to estimate the degree of disorder in graphene.

\section{SUMMARY}

Raman spectroscopy in 2D materials research has much wider applications than in most other materials. In addition to structural properties, electrical properties and band structures affect the measured Raman spectrum of most 2D materials. By analyzing Raman spectra, one can obtain information on the number of layers, stacking orders, defects, crystallographic orientation, doping density, etc. In TMDCs, resonance with excitonic states results in anomalous effects, which reflect the peculiar nature of 2D materials.

\section{CONFLICT OF INTEREST}

No potential conflict of interest relevant to this article was reported.

\section{REFERENCES}

Blake P, Hill E W, Castro Neto A H, Novoselov K S, Jiang D, Yang R, Booth T J, and Geim A K (2007) Making graphene visible. Appl. Phys. Lett. 91, 063124.

Cançado L G, Jorio A, Ferreira E H M, Stavale F, Achete C A, Capaz R B, Moutinho M V O, Lombardo A, Kulmala T S, and Ferrari A C (2011) Quantifying defects in graphene via Raman spectroscopy at different excitation energies. Nano Lett. 11, 3190-3196.

Carvalho B R, Malard L M, Alves J M, Fantini C, and Pimenta M A (2015) Symmetry-dependent exciton-phonon coupling in 2D and bulk $\mathrm{MoS}_{2}$ observed by resonance Raman scattering. Phys. Rev. Lett. 114, 136403.

Castro Neto A H, Guinea F, Peres N M R, Novoselov K S, and Geim A K (2009) The electronic properties of graphene. Rev. Mod. Phys. 81, 109-162.

Chakraborty B, Bera A, Muthu D V S, Bhowmick S, Waghmare U V, and Sood A K (2012) Symmetry-dependent phonon renormalization in monolayer $\mathrm{MoS}_{2}$ transistor. Phys. Rev. B 85, 161403.

Chakraborty B, Matte H S S R, Sood A K, and Rao C N R (2013) Layerdependent resonant Raman scattering of a few layer $\mathrm{MoS}_{2}$. J. Raman Spectrosc. 44, 92-96.

Chen S Y, Zheng C, Fuhrer M S, and Yan J (2015) Helicity-resolved Raman scattering of $\mathrm{MoS}_{2}, \mathrm{MoSe}_{2}, \mathrm{WS}_{2}$, and $\mathrm{WSe}_{2}$ atomic layers. Nano Lett. 15, 2526-2532.

Cong C, Yu T, Sato K, Shang J, Saito R, Dresselhaus G F, and Dresselhaus M S (2011) Raman characterization of ABA- and ABC-stacked trilayer graphene. ACS Nano 5, 8760-8768.

Conley H J, Wang B, Ziegler J I, Haglund R F, Pantelides S T, and Bolotin K I (2013) Bandgap engineering of strained monolayer and bilayer $\mathrm{MoS}_{2}$. Nano Lett. 13, 3626-3630.

Dean C R, Young A F, Meric I, Lee C, Wang L, Sorgenfrei S, Watanabe K, Taniguchi T, Kim P, Shepard K L, and Hone J (2010) Boron nitride substrates for high-quality graphene electronics. Nat. Nanotechnol. 5, 722-726. del Corro E, Terrones H, Elias A, Fantini C, Feng S, Nguyen M A, Mallouk T E, Terrones M, and Pimenta M A (2014) Excited excitonic states in 1L, $2 \mathrm{~L}, 3 \mathrm{~L}$, and bulk $\mathrm{WSe}_{2}$ observed by resonant Raman spectroscopy. ACS Nano 8, 9629-9635.

Dresselhaus M S, Jorio A, Souza Filho A G, and Saito R (2010) Defect characterization in graphene and carbon nanotubes using Raman spectroscopy. Philos. Trans. R. Soc. A Math. Phys. Eng. Sci. 368, 5355-5377.

Eckmann A, Felten A, Mishchenko A, Britnell L, Krupke R, Novoselov K S, and Casiraghi $C$ (2012) Probing the nature of defects in graphene by Raman spectroscopy. Nano Lett. 12, 3925-3930.

Fan J H, Gao P, Zhang A M, Zhu B R, Zeng H L, Cui X D, He R, and Zhang Q M (2014) Resonance Raman scattering in bulk $2 \mathrm{H}-\mathrm{MX} 2$ (M = Mo, W; $\mathrm{X}=\mathrm{S}, \mathrm{Se}$ ) and monolayer $\mathrm{MoS}_{2}$. J. Appl. Phys. 115, 053527.

Ferrari A C and Basko D M (2013) Raman spectroscopy as a versatile tool for studying the properties of graphene. Nat. Nanotechnol. 8, 235246.

Ferrari A C, Meyer J C, Scardaci V, Casiraghi C, Lazzeri M, Mauri F, Piscanec S, Jiang D, Novoselov K S, Roth S, and Geim A K (2006) Raman spectrum of graphene and graphene layers. Phys. Rev. Lett. 97, 187401.

Geim A K and Novoselov K S (2007) The rise of graphene. Nat. Mater. 6 , 183-191.

Gołasa K, Grzeszczyk M, Leszczyński P, Faugeras C, Nicolet A A L, Wysmołek A, Potemski M, and Babiński A (2014) Multiphonon resonant Raman scattering in MoS $_{2}$. Appl. Phys. Lett. 104, 092106.

Jegal S, Hao Y, Yoon D, Ruoff R S, Yun H, Lee S W, and Cheong H (2013) Crystallographic orientation of early domains in CVD graphene studied by Raman spectroscopy. Chem. Phys. Lett. 568-569, 146150.

Klots A R, Newaz A K M, Wang B, Prasai D, Krzyzanowska H, Lin J, Caudel D, Ghimire N J, Yan J, Ivanov B L, Velizhanin K A, Burger A, Mandrus D G, Tolk N H, Pantelides S T, and Bolotin K I (2014) Probing 
excitonic states in suspended two-dimensional semiconductors by photocurrent spectroscopy. Sci. Rep. 4, 6608.

Lee C, Yan H, Brus L E, Heinz T F, Hone J, and Ryu S (2010) Anomalous lattice vibrations of single- and few-layer $\mathrm{MoS}_{2}$. ACS Nano 4, 26952700.

Lee J E, Ahn G, Shim J, Lee Y S, and Ryu S (2012) Optical separation of mechanical strain from charge doping in graphene. Nat. Commun. $\mathbf{3}$, 1024.

Lee J U, Park J, Son Y W, and Cheong H (2015) Anomalous excitonic resonance Raman effects in few-layered $\mathrm{MoS}_{2}$. Nanoscale 7, 32293236.

Li L, Yu Y, Ye G J, Ge Q, Ou X, Wu H, Feng D, Chen X H, and Zhang Y (2014) Black phosphorus field-effect transistors. Nat. Nanotechnol. 9, 372377.

Li S L, Miyazaki H, Song H, Kuramochi H, Nakaharai S, and Tsukagoshi K (2012) Quantitative Raman spectrum and reliable thickness identification for atomic layers on insulating substrates. ACS Nano $\mathbf{6}$, 7381-7388.

Liu H, Neal A T, Zhu Z, Luo Z, Xu X, Tománek D, and Ye P D (2014) Phosphorene: an unexplored 2D semiconductor with a high hole mobility. ACS Nano 8, 4033-4041.

Livneh T and Spanier J E (2015) A comprehensive multiphonon spectral analysis in $\mathrm{MoS}_{2}$. 2D Mater. 2, 035003.

Lui C H, Li Z, Chen Z, Klimov P V, Brus L E, and Heinz T F (2011) Imaging stacking order in few-layer graphene. Nano Lett. 11, 164-169.

Luo X, Zhao Y, Zhang J, Toh M, Kloc C, Xiong Q, and Quek S Y (2013) Effects of lower symmetry and dimensionality on Raman spectra in two-dimensional WSe $\mathrm{W}_{2}$. Phys. Rev. B 88, 195313.

Mak K F, Lee C, Hone J, Shan J, and Heinz T F (2010) Atomically thin $\mathrm{MoS}_{2}$ : a new direct-gap semiconductor. Phys. Rev. Lett. 105, 136805.

Mignuzzi S, Pollard A J, Bonini N, Brennan B, Gilmore I S, Pimenta M A, Richards D, and Roy D (2015) Effect of disorder on Raman scattering of single-layer $\mathrm{MoS}_{2}$. Phys. Rev. B 91, 195411

Mohiuddin T M G, Lombardo A, Nair R R, Bonetti A, Savini G, Jalil R, Bonini N, Basko D M, Galiotis C, Marzari N, Novoselov K S, Geim A K, and Ferrari A C (2009) Uniaxial strain in graphene by Raman spectroscopy: G peak splitting, Grüneisen parameters, and sample orientation. Phys. Rev. B 79, 205433.

Molina-Sánchez A and Wirtz L (2011) Phonons in single-layer and fewlayer $\mathrm{MoS}_{2}$ and $\mathrm{WS}_{2}$. Phys. Rev. B 84, 155413.

Nguyen T A, Lee J U, Yoon D, and Cheong H (2014) Excitation energy dependent Raman signatures of ABA- and ABC-stacked few-layer graphene. Sci. Rep. 4, 4630.

Novoselov K S, Geim A K, Morozov S V, Jiang D, Zhang Y, Dubonos S V, Grigorieva I V, and Firsov A A (2004) Electric field effect in atomically thin carbon films. Science 306, 666-669.

Pimenta M A, del Corro E, Carvalho B R, Fantini C, and Malard L M (2015) Comparative study of Raman spectroscopy in graphene and $\mathrm{MoS}_{2}$ type transition metal dichalcogenides. Acc. Chem. Res. 48, 41-47.
Qiu D Y, da Jornada F H, and Louie S G (2013) Optical spectrum of MoS: many-body effects and diversity of exciton states. Phys. Rev. Lett. 111, 216805.

Scheuschner N, Ochedowski O, Schleberger M, and Maultzsch J (2012) Resonant Raman profiles and $\mu$-photoluminescence of atomically thin layers of molybdenum disulfide. Phys. Status Solidi 249, 2644 2647.

Staiger M, Gillen R, Scheuschner N, Ochedowski O, Kampmann F, Schleberger M, Thomsen C, and Maultzsch J (2015) Splitting of monolayer out-of-plane $\mathrm{A}_{1}{ }^{\prime}$ Raman mode in few-layer $\mathrm{WS}_{2}$. Phys. Rev. $B$ 91, 195419.

Tan P H, Han W P, Zhao W J, Wu Z H, Chang K, Wang H, Wang Y F, Bonini N, Marzari N, Pugno N, Savini G, Lombardo A, and Ferrari A C (2012) The shear mode of multilayer graphene. Nat. Mater. 11, 294-300.

Tonndorf P, Schmidt R, Böttger P, Zhang X, Börner J, Liebig A, Albrecht M, Kloc C, Gordan O, Zahn D R T, Michaelis de Vasconcellos S, and Bratschitsch R (2013) Photoluminescence emission and Raman response of monolayer $\mathrm{MoS}_{2}, \mathrm{MoSe}_{2}$, and $\mathrm{WSe}_{2}$. Opt. Express 21, 4908-4916.

Wang Q H, Kalantar-Zadeh K, Kis A, Coleman J N, and Strano M S (2012) Electronics and optoelectronics of two-dimensional transition metal dichalcogenides. Nat. Nanotechnol. 7, 699-712.

Wang Y, Cong C, Qiu C, and Yu T (2013) Raman spectroscopy study of lattice vibration and crystallographic orientation of monolayer $\mathrm{MoS}_{2}$ under uniaxial strain. Small 9, 2857-2861.

Yan J, Zhang Y, Kim P, and Pinczuk A (2007) Electric field effect tuning of electron-phonon coupling in graphene. Phys. Rev. Lett. 98, 166802

Yoon D, Moon H, Cheong H, Choi J, Choi J, and Park B (2009a) Variations in the Raman spectrum as a function of the number of graphene layers. J. Korean Phys. Soc. 55, 1299-1303.

Yoon D, Moon H, Son Y W, Choi J S, Park B H, Cha Y H, Kim Y D, and Cheong $\mathrm{H}$ (2009b) Interference effect on Raman spectrum of graphene on $\mathrm{SiO}_{2} / \mathrm{Si}$. Phys. Rev. B 80, 125422.

Yoon D, Son Y W, and Cheong H (2011) Strain-dependent splitting of the double-resonance raman scattering band in graphene. Phys. Rev. Lett. 106, 155502

Zhang X, Han W P, Wu J B, Milana S, Lu Y, Li Q Q, Ferrari A C, and Tan P H (2013) Raman spectroscopy of shear and layer breathing modes in multilayer $\mathrm{MoS}_{2}$. Phys. Rev. B 87, 115413.

Zhang X, Qiao X F, Shi W, Wu J B, Jiang D S, and Tan P H (2015) Phonon and Raman scattering of two-dimensional transition meta dichalcogenides from monolayer, multilayer to bulk material. Chem. Soc. Rev. 44, 2757-2785.

Zhao W, Ghorannevis Z, Amara K K, Pang J R, Toh M, Zhang X, Kloc C, Tan P H, and Eda G (2013a) Lattice dynamics in mono- and few-layer sheets of $\mathrm{WS}_{2}$ and $\mathrm{WSe}_{2}$. Nanoscale 5, 9677.

Zhao Y, Luo X, Li H, Zhang J, Araujo P T, Gan C K, Wu J, Zhang H, Quek S Y, Dresselhaus M S, and Xiong Q (2013b) Interlayer breathing and shear modes in few-trilayer $\mathrm{MoS}_{2}$ and $\mathrm{WSe}_{2}$. Nano Lett. 13, 1007 1015. 